\title{
GMR
}

\section{Correlation analysis between the delayed rectifier potassium channel KCNE1 (G38S) polymorphism and atrial fibrillation among the senior Uygur population in Xinjiang}

\author{
N. Wugeti*, G. Yu-Jun*, S. Juan and A. Mahemuti \\ Department of Heart Failure, The First Affiliated Hospital of Xinjiang Medical University, \\ Urumqi, Xinjiang, China \\ *These authors contributed equally to this study. \\ Corresponding author: A. Mahemuti \\ E-mail: 454726435@qq.com \\ Genet. Mol. Res. 14 (4): 15906-15912 (2015) \\ Received August 3, 2015 \\ Accepted October 29, 2015 \\ Published December 7, 2015 \\ DOI http://dx.doi.org/10.4238/2015.December.7.1
}

ABSTRACT. Current resources to support genetic screening among the Uygur population in Xinjiang territory for atrial fibrillation (AF) have not been well established and large-scale epidemiological analyses are needed. Using patients from the Xinjiang Uygur population as subjects, and the delayed rectifier potassium channel KCNE1 and its associated polymorphism G38S (rs1805127) as the candidate gene, we analyzed the correlation between the G38S polymorphism and AF among the senior Uygur population in Xinjiang Province. Peripheral blood from AF Uygur patients (patient group) or non-AF Uygur patients (control group) from Xinjiang territory was collected (70 patients each). DNA was purified and tested by polymerase chain reaction-restriction fragment length polymorphism for the genotype and allelic distribution of KCNE1 (G38S). Correlation analysis between $\mathrm{AF}$ and multiple health-related factors was performed by logistic regression. Among patients with the KCNE1 G38S polymorphism, the genotypes AA, AG, and GG were present at frequencies of 17.14, 27.14, 
and $55.71 \%$, respectively, in the patient group, compared with $24.29,50$, and $25.71 \%$, respectively, in the control group. The difference between these two groups was shown to be statistically significant $(P<0.05)$, and the frequency of the $G$ allele was significantly higher in the patient group $(P<0.05)$. Logistic regression showed that the $G G$ genotype is correlated with the incidence of $A F$ in Uygur seniors $(P<0.05)$. The incidence of AF among the senior Uygur population in Xinjiang territory was correlated with the KCNE1 (G38S) polymorphism, which may be an independent risk factor for Uygur AF patients.

Key words: Atrial fibrillation; Gene polymorphism; KCNE1 (G38S); Uygur population

\section{INTRODUCTION}

Atrial fibrillation (AF) is a severe progressive arrhythmia commonly seen in clinical settings. The rate of incidence of AF in Chinese adults has been reported to be $0.77 \%$ (Zhou et al., 2004). With recent developments in molecular biology techniques, exploration of the pathology of AF at the genetic level has become an emerging topic in current AF studies. Current resources to support genetic screening among the Uygur population in Xinjiang territory for AF have not been well established and large-scale epidemiological analyses are needed. Using patients from the Xinjiang Uygur population as subjects, and the delayed rectifier potassium channel KCNE1 and its associated polymorphism G38S (rs1805127) as the candidate gene, we analyzed the differential distribution of genotypes and allele frequencies among patients with or without AF. The correlation between the KCNE1 (G38S, rs1805127) gene polymorphism and AF incidence among Uygur seniors in Xinjiang is further discussed to provide detailed genetic evidence for molecular epidemiological studies.

\section{MATERIAL AND METHODS}

\section{Subjects}

Forty-five Uygur patients diagnosed with AF between August 2010 and August 2011 at the First Teaching Hospital of Xinjiang Medical University were enrolled in this study. Further random samples from the Uygur AF database (primarily built by the Xinjiang Uygur Autonomous Region Natural Science Foundation "Current Xinjiang AF Epidemiology" group using a flow survey in Kashi and Turpan) were added to form the Uygur AF patient group. The group comprised 48 males and 22 females, whose ages ranged from 60 to 85 years with an average age of $65.26 \pm 4.17$ years. Seventy non-AF patients, hospitalized during the same period, were selected as the control group. The control group comprised 48 males and 22 females with an average age of $62.25 \pm 7.35$ years. None of the electrocardiograms (including Holter) of the patients in the control group showed symptoms of AF. There were no statistically significant differences in gender or age between the two groups.

\section{General participant information}

This study was approved by the ethics committee of the First Teaching Hospital of Xinjiang Medical University. Informed consent was obtained from each participant. A survey table was used 
to record general information on the subjects and major risk factors for cardiovascular diseases and $A F$, including hypertension, coronary heart disease, smoking, alcohol consumption, left atrium diameter, and left ventricular ejection fraction. Survey methods and quality control were executed as follows: internationally standardized survey methods were used to develop a unified design and plan for an epidemiological investigation centered on AF. A questionnaire and a physical examination were included in the survey. The questionnaire included general information, occupation, workload, individual AF history, family history, and information about smoking and alcohol consumption. The physical examination included production of an electrocardiogram, and measurement of blood pressure, heart rate, height, body mass, and waist, abdominal, and hip circumferences.

Selection criteria for the AF group were as follows (Zhang and Xia, 2006): 1) the current electrocardiogram indicated AF; 2) patients were diagnosed by the physician as having AF and had a previous electrocardiogram or Holter records that led to the diagnosis of AF; and 3) a previous electrocardiogram or Holter records indicated the presence of AF. Hypertension was diagnosed based on the criteria from the Guidelines for the Management of Hypertension published in 1999.

\section{Experimental procedures}

Peripheral blood ( $5 \mathrm{~mL}$ ) was treated using $0.5 \mathrm{M}$ ethylenediaminetetraacetic acid (EDTA). High salt precipitation was performed to extract peripheral white blood cell DNA, which was then dissolved in Tris-EDTA buffer and preserved at $-20^{\circ} \mathrm{C}$. A UV spectrophotometer was used to measure the DNA concentration of the samples, which were then diluted for further assays. The DNA was then tested for the KCNE1 (G38S, rs1805127) gene polymorphism. The polymerase chain reaction (PCR) was used to amplify KCNE1-containing polymorphic fragments. Restriction enzymes were used to identify genotypes. Primers used to amplify the gene segment containing the single nucleotide polymorphism in KCNE1 (rs1805127) were as follows: forward $\left(5^{\prime} \rightarrow 3^{\prime}\right)$ : GTGACGCCCTTTC TGACCAA; and reverse $\left(3^{\prime} \rightarrow 5^{\prime}\right)$ : CCAGATGGT TTTCAACGACA. The PCR product was 318 base pairs (bp) in length. The KCNE1 G38S polymorphism is a mutation from A to $G$ at the 112th residue, resulting in a Ser-to-Gly (S-G) polymorphism at the 38th amino acid residue of the encoded protein. After digestion by MspA1I, three genotypes could be visualized by gel electrophoresis. If the nucleotide $G$ is present at the allele, it forms a restriction digest site for the enzyme MspA1I, while the restriction digest site is not present when the A allele is found. Therefore, two bands of 232 and 86 bp were observed for the GG genotype, while three bands (318, 232, and $86 \mathrm{bp}$ ) were present for the AG genotype. A single $318 \mathrm{bp}$ band was observed for the AA genotype. Genotyping was carried out by electrophoresis. After PCR amplification, $5 \mu \mathrm{L}$ PCR product was loaded onto a $1.5 \%$ agarose electrophoresis gel with a 100 bp DNA molecular marker. The PCR product was then stored until restriction digestion.

\section{Statistical analysis}

SPSS 17.0 was employed for statistical analysis. SHEsis software was used to perform the Hardy-Weinberg equilibrium test based on genotype distribution to confirm whether the samples were representative of the population. If the distribution passed the Hardy-Weinberg equilibrium test, then the recorded data from each group were recorded as means \pm standard deviation. Twogroup comparisons of these were analyzed by the $t$-test. Two-group comparisons of genotypes and allele frequencies were analyzed by the $\mathrm{X}^{2}$-test. The integrative evaluation between AF and multiple 
factors was analyzed using multiple factor logistic regression analysis. $\mathrm{P}<0.05$ was used as the cutoff for statistical significance.

\section{RESULTS}

\section{General comparison}

As shown in Table 1, no significant difference was observed in smoking or alcohol use between the patient and control groups $(P>0.05)$, while coronary heart disease and hypertension were present at significantly higher rates within the AF group when compared with the control group $(P<0.05$ and 0.01 , respectively). Statistically significant differences were also seen in the left atrium diameter and left ventricular ejection fraction values $(P<0.01)$.

Table 1. General comparison between the control and patient groups.

\begin{tabular}{lcc}
\hline Potential risk factor & Patient group $(\mathrm{N}=70)$ & Control group $(\mathrm{N}=70)$ \\
\hline LAD (means $\pm \mathrm{SD}, \mathrm{mm})$ & $36.76 \pm 2.36^{\star *}$ & $29.37 \pm 2.54$ \\
LVEF (means \pm SD, \%) & $60.23 \pm 2.50^{* *}$ & $65.68 \pm 2.75$ \\
Coronary heart disease (N, \%) & $49(70)^{*}$ & $18(25,71)$ \\
Hypertension (N, \%) & $53(75,71)^{\star *}$ & $17(24,29)$ \\
Smoking (N, \%) & $22(31,43)$ & $18(25,71)$ \\
Alcohol consumption (N, \%) & $8(11,43)$ & $10(14,29)$ \\
\hline
\end{tabular}

$\mathrm{LAD}=$ left atrium diameter; LVEF = left ventricular ejection fraction. ${ }^{*} \mathrm{P}<0.05,{ }^{* *} \mathrm{P}<0.01$ vs control group.

\section{Comparison of KCNE1 (G38S) genotypes and allelic frequencies}

As shown in Table 2, the frequencies of the genotypes AA, AG, and GG in the patient group were $17.14,27.14$, and $55.71 \%$, respectively, indicating a dominance of the GG genotype. In the control group, the frequencies of the genotypes AA, AG, and GG were 24.29 , 50, and $25.71 \%$, respectively, showing a dominance of the AG genotype. This difference between the two groups was statistically significant $\left(X^{2}=6.435, P<0.05\right)$. The major allele was $G$ for both the patient and control groups, although individuals in the control group had a higher frequency of the $G$ allele $\left(X^{2}\right.$ $=6.560, \mathrm{P}<0.05)$.

Table 2. KCNE1 (G38S) genotype distribution and allele frequency (\%).

\begin{tabular}{|c|c|c|c|c|c|c|c|c|c|}
\hline & \multicolumn{3}{|c|}{ Genotype } & \multirow[t]{2}{*}{$x^{2}$} & \multirow[t]{2}{*}{$P$} & \multicolumn{2}{|c|}{ Allele } & \multirow[t]{2}{*}{$x^{2}$} & \multirow[t]{2}{*}{$\mathrm{P}$} \\
\hline & AA & $A G$ & GG & & & A & G & & \\
\hline Patient group & $12(17.14)$ & $19(27.14)$ & $39(55.71)$ & 6.435 & 0.026 & $43(30.71)$ & $97(69.29)$ & 6.560 & 0.033 \\
\hline Control group & $17(24.29)$ & $35(50)$ & $18(25.71)$ & & & 69 (49.29) & $71(50.71)$ & & \\
\hline
\end{tabular}

\section{Correlation analysis between KCNE1 (G38S) polymorphism and AF}

Multiple factor logistic regression analysis was used to analyze the correlation between the KCNE1 (G38S) polymorphism and AF, and the selection of model variables was based on the forward Wald method. As shown in Table 3, risk factors for AF among the senior Uygur AF population included coronary heart disease and hypertension. After controlling for the mixed influence from 
hypertension, coronary heart disease, smoking, and alcohol consumption, a statistically significant difference between the incidence of the KCNE1 (G38S) GG genotype was observed between the patient and control groups $(P<0.05)$. Individuals carrying the $G G$ genotype had a 1.526 times higher risk of developing AF than non-carriers.

\begin{tabular}{|c|c|c|c|c|c|}
\hline Risk factors & $\beta$-value & SE & Wald $X^{2}$ & OR & $\mathrm{P}$ \\
\hline Coronary heart disease & 0.530 & 0.138 & 11.37 & 1.650 & $<0.01$ \\
\hline Hypertension & 0.648 & 0.155 & 14.56 & 1.975 & $<0.01$ \\
\hline Smoking & 0.150 & 0.326 & 4.96 & 1.228 & 0.106 \\
\hline Alcohol consumption & 0.146 & 0.377 & 4.30 & 1.256 & 0.112 \\
\hline \multicolumn{6}{|l|}{ KCNE1 (G38S) } \\
\hline AA & 0.122 & 0.230 & 3.98 & 1.245 & 0.240 \\
\hline GG & 0.488 & 0.120 & 10.65 & 1.526 & $<0.01$ \\
\hline$A G$ & 0.132 & 0.205 & 3.25 & 1.130 & 0.338 \\
\hline
\end{tabular}

$\mathrm{SE}=$ standard error; $\mathrm{OR}=$ odds ratio.

\section{DISCUSSION}

Brugada et al. (1997) reported 10q22-q24 as the first AF-associated sites, demonstrating a genetic basis for AF. Among multiple AF candidate genes, in addition to ion channel-associated genes, several non-ion channel-associated genes showed polymorphisms associated with AF incidence. Most AF-associated genes trigger this disease by structurally and functionally altering different ion channels in the atrial myocardium. Slow delayed rectifier potassium current $\left(\mathrm{I}_{\mathrm{Ks}}\right)$ and rapid delayed rectifier potassium current $\left(\mathrm{I}_{\mathrm{Kr}}\right)$ are the major repolarizing currents in cardiomyocytes. It has been suggested that elevated function of these ion channels results in faster repolarization as well as shorter action potential and a shorter effective refractory period. This may be a contributor to the onset and development of AF. The voltage-gated potassium channels (Kv channels) include delayed rectifier potassium channels and transient outward potassium channels (Ito). Delayed rectifier potassium channels can be further classified as $I_{\mathrm{Ks}}$ and $I_{\mathrm{Kr}}$. KCNQ1 and KCNE1 encode the $\alpha$ - and $\beta$-subunits, respectively, of IKs (Dai et al., 2010).

Genetic mutations in KCNE family members can alter the $\mathrm{I}_{\mathrm{Ks}}$ and $\mathrm{I}_{\mathrm{Kr}}$ current density and channel dynamics, leading to changes in action potential timing and effective refractory period. Together, these changes may result in the onset or development of AF. Fatini et al. (2006) found that in non-valvular Caucasian AF patients the $38 \mathrm{G}$ allele of the minK gene is associated with AF. Schreieck et al. (2004) reported that the C825T polymorphism is associated with sporadic AF, while patients with the TT genotype show a higher $I_{K 1}$ current density. This result also suggests that changes in the ion channel inward rectifying function are associated with familiar and sporadic AF (Schreieck et al., 2004). Ackerman et al. (2003) found two mutations in the Asian population, namely G38S (112G>A, 16.4\% of heterozygote frequency) and D85N (253G>A, $0.7 \%$ of heterozygote frequency). Lai et al. (2002) showed that the $38 \mathrm{G}$ allele of KCNE1 had a higher risk of inducing AF than the 38S allele. KCNE1 38G leads to decreased $\mathrm{I}_{\mathrm{Ks}}$ density and unstable atrial action potential repolarization, which eventually contributes to AF. Ehrlich et al. (2005) investigated the function of minK $38 \mathrm{G}$ and suggested that reduced membrane expression of KCNQ1 and reduced $\mathrm{I}_{\mathrm{Ks}}$ current can be induced by minK38G. Recently, it has been shown that the polymorphism of minK38G is associated with sporadic AF as a genetic risk, with GG genotype carrying a greater risk than GS or SS (Prystupa et al., 2006). Our study demonstrated a difference in the distribution of the KCNE1 
(G38S) genotypes between Uygur AF and non-AF patients. While $G$ was the major allele for both groups, AF patients had a higher frequency of $G$ alleles, which suggests that carriers of this allele have a higher risk of developing AF.

AF shows differential incidence rates among different ethnic groups. In 2006, the AF management guidelines from the American College of Cardiology, the American Heart Association, and the European Society of Cardiology suggested that black individuals have half the rate of incidence of white individuals, indicating racial differences in the mechanism of AF development. Two studies have indicated that sporadic AF may be associated with certain genetic or molecular changes in the body. Olson et al. (2005) demonstrated that changes in SCN5A can lead to dilated cardiomyopathy and AF. Chen et al. (2007) further indicated that SCN5A polymorphisms were observed among Asian, Latin American, Caucasian, and African American populations, suggesting that prevention of AF in these four groups should take place at an earlier time. Mu et al. (2008) performed a comparative study between Han and Uygur AF patients and found racial differences in clinical features including age of incidence, cause, left ventricular diameter, and valvular heart disease. Yao et al. (2011) showed that the KCNE1 gene (rs1805127) polymorphism increases the AF risk in Xinjiang Uygur individuals. Our study enhanced the resources for future epidemiological studies of the Xinjiang Uygur AF population, providing a fundamental basis for genetically individualized and geographically localized therapy for the future.

\section{Conflicts of interest}

The authors have no conflicts of interest.

\section{ACKNOWLEDGMENTS}

Research supported by grant \#2012211A064 from the Natural Science Foundation of Xinjiang Uygur Autonomous Region. Ethical approval was given by the medical ethics committee of the People's Hospital of Xinjiang Uygur Nationality Autonomous Region.

\section{REFERENCES}

Ackerman MJ, Tester DJ, Jones GS, Will ML, et al. (2003). Ethnic differences in cardiac potassium channel variants: implications for genetic susceptibility to sudden cardiac death and genetic testing for congenital long QT syndrome. Mayo Clin. Proc. 78: 1479-1487.

Brugada R, Tapscott T, Czernuszewicz GZ, Marian AJ, et al. (1997). Identification of a genetic locus for familial atrial fibrillation. N. Engl. J. Med. 336: 905-911.

Chen LY, Ballew JD, Herron KJ, Rodeheffer RJ, et al. (2007). A common polymorphism in SCN5A is associated with lone atrial fibrillation. Clin. Pharmacol. Ther. 81: 35-41.

Dai Z, Liu J, Wang Y, Zeng Q, et al. (2010). Gene and protein expression of Kir3.4 of KAch channel in chronic human atrial fibrillation. Shandong Med. 50: 1-3.

Ehrlich JR, Zicha S, Coutu P, Hébert TE, et al. (2005). Atrial fibrillation-associated minK38G/S polymorphism modulates delayed rectifier current and membrane localization. Cardiovasc. Res. 67: 520-528.

Fatini C, Sticchi E, Genuardi M, Sofi F, et al. (2006). Analysis of minK and eNOS genes as candidate loci for predisposition to non-valvular atrial fibrillation. Eur. Heart J. 27: 1712-1718.

Lai LP, Su MJ, Yeh HM, Lin JL, et al. (2002). Association of the human Minx gene 38G allele with atrial fibrillation: evidence of possible genetic control on the pathogenesis of atrial fibrillation. Am. Heart J. 144: 485-490.

$\mathrm{Mu} \mathrm{H}$, Lu W and Ma Y (2008). The comparison of clinical features in atrial fibrillation between the Uygurs and Hans patients. Chin. J. Cardiac Pacing Electrophysiol. 22: 216-218. 
Olson TM, Michels VV, Ballew JD, Reyna SP, et al. (2005). Sodium channel mutations and susceptibility to heart failure and atrial fibrillation. JAMA 293: 447-454.

Prystupa A, Dzida G, Myśliński W, Małaj G, et al. (2006) Mink gene polymorphism in the pathogenesis of lone atrial fibrillation. Kardiol. Pol. 64: 1205-1211.

Schreieck J, Dostal S, von Beckerath N, Wacker A, et al. (2004). C825T polymorphism of the G-protein beta3 subunit gene and atrial fibrillation: association of the TT genotype with a reduced risk for atrial fibrillation. Am. Heart J. 148: 545-550.

Yao J, Ma YT, Xie X, Liu F, et al. (2011). Association of rs1805127 polymorphism of KCNE1 gene with atrial fibrillation in Uigur population of Xinjiang. Zhonghua Yi Xue Yi Chuan Xue Za Zhi 28: 436-440.

Zhang D and Xia X (2006). Clinical analysis of elderly patients with atrial fibrillation. Practical Geriatrics 20: 142-143.

Zhou ZQ, Hu DY, Chen J, Zhang RH, et al. (2004). An epidemiological survey of atrial fibrillation in China. Zhonghua Nei Ke Za Zhi 43: 491-494. 